\title{
Energy-efficient capture of stochastic events based on on-line scheduling scheme
}

\author{
Xiao Fu ${ }^{1 *}$ D, Zongmao Cheng ${ }^{2}$ and Hao $\operatorname{Tan}^{2}$
}

\author{
* Correspondence: fuxiao@hdu.edu. \\ $\mathrm{cn}$ \\ ${ }^{1}$ Zhejiang Informatization \\ Development Institute, Hangzhou \\ Dianzi University, Hangzhou 310018, \\ China \\ Full list of author information is \\ available at the end of the article
}

\begin{abstract}
We improve the off-line scheduling scheme of existing wireless sensor network. Firstly, we introduce Bayesian statistical method in synchronous wireless sensor network. Then, we let duration and interval, the reflection of characteristics of stochastic events, obey exponential distribution. Next, we make Bayes posterior estimation on parameter. Based on Bayesian estimate, we obtain the analytical solution of the capture probability of stochastic events and sensor energy efficiency of capture events. Finally, we propose an on-line scheduling scheme for synchronous wireless sensor networks. This paper compares and analyzes the simulation experiments in on-line scheduling scheme and off-line scheduling scheme, and the results show that compared with off-line scheduling scheme with constant distribution parameter values, on-line scheduling scheme can effectively reduce the probability of missing stochastic events and increase the probability of capturing events, further save energy consumption of wireless sensor network, and extend network lifetime.

Keywords: Synchronous wireless sensor networks, Stochastic events, Bayesian statistical method, On-line scheduling scheme
\end{abstract}

\section{Introduction}

The Internet of Things (IoT), the currently most popular research area, refers to realtime monitoring and connecting objects through various sensor devices and technologies to collect event information, and then having the access to the network, it finally realizes the ubiquitous connection between object and object as well as object and people. Wireless sensor networks (WSN) is the key technology for the development of IoT $[1,2]$. WSN is composed of hundreds of small sensors, and each sensor is capable of perceiving, collecting, calculating, and forwarding event information [3]. Events do not always occur and appear randomly, but their randomness can be described by two random variables, the event staying time at Point of Interest (PoI) and the interval between events. If the event duration is short but the interval is long, the sensor is always in active state, which is bound to cause waste energy. Therefore, how to schedule the active and inactive state of the sensor in WSN saves the energy of sensor network as

(c) The Author(s). 2020 Open Access This article is licensed under a Creative Commons Attribution 4.0 International License, which permits use, sharing, adaptation, distribution and reproduction in any medium or format, as long as you give appropriate credit to the original author(s) and the source, provide a link to the Creative Commons licence, and indicate if changes were made. The images or other third party material in this article are included in the article's Creative Commons licence, unless indicated otherwise in a credit line to the material. If material is not included in the article's Creative Commons licence and your intended use is not permitted by statutory regulation or exceeds the permitted use, you will need to obtain permission directly from the copyright holder. To view a copy of this licence, visit http://creativecommons.org/licenses/by/4.0/. 
much as possible, and extending sensor network lifetime is a hot issue in current research.

At present, the sleeping scheduling issue in WSN can be divided into three categories: (1) randomness without considering stochastic events [4-6], that is, to find nonoverlapping subsets covered by sensors and let them work alternately so as to save energy and extend network lifetime. (2) Considering the randomness of stochastic events interval but not considering the randomness of duration of stochastic events [7-10], the sensor scheduling scheme is designed only based on the randomness of interval between events arrival. (3) Considering the randomness of interval between stochastic events arrival as well as the randomness of duration of stochastic events [11-14], the sensor inactive state scheduling problem is transformed into an energy-oriented optimization problem, and the algorithm is designed to solve the issue. In the latter two categories, it is usually assumed that the interval and duration of stochastic events are subject to exponential distribution, where the distribution parameter is a given constant, and the inactive period of sensor is also constant. This mode is called an off-line scheduling scheme.

From a statistical point of view, the distribution parameter given by the off-line scheduling scheme are estimated based on historical data, and once this parameter is given at a fixed value, it remains unchanged regardless of posterior sample. However, in practical applications, event fluctuations may occur due to various reasons. For example, samples obtained in different time periods may be generated by exponential distributions with different distribution parameters. This requires the scheduling scheme of sensor to adjust distribution parameters based on the sample; otherwise, huge errors would occur. Therefore, based on the research made by He et al. [15], we study on-line scheduling issue of sensors in synchronous WSN and propose a real-time adjustable sensor inactive period scheduling scheme to make it more in line with randomness of stochastic event, more adaptable, and further save energy consumption of network.

The main research contents of this paper are as follows: (1) Firstly, by introducing Bayes statistics method to deal with previous off-line scheduling problem in synchronous WSN. Bayesian statistics method is used to estimate interval parameters of stochastic events and distribution parameters of duration and Bayes estimated value with real-time adjustment is obtained. (2) Then, the relationship between capture probability of stochastic events, the energy efficiency of sensor to capture stochastic event, and Bayesian estimated value of distribution parameter is discussed, and the relation between periodic schedule and Bayesian estimated value of distribution parameter is obtained. (3) Finally, the sensor periodic schedule that can be adjusted in real time in synchronous WSN is proposed, that is, an on-line scheduling scheme.

The contributions of this paper can be summarized as follows: (1) Since Bayesian estimation can be iteratively repeated, the method in this paper can be used to study the periodic sensor scheduling with real-time adjustment. (2) By increasing Bayesian estimation of distribution parameters, the randomness of stochastic events can be more accurately characterized. (3) By conducting simulation experiments, compared with previous off-line scheduling scheme, it shows that on-line scheduling scheme of sensor sleep cycle can effectively save energy and extend network lifetime.

The rest of the paper is as follows: the second section is related work; the third section is model and hypothesis, introducing Bayesian estimation of exponential 
distribution parameters; the fourth section analyzes the event capture performance under the Bayesian estimation of distribution parameters; the fifth section studies online scheduling scheme; the sixth section is simulation experiment; and the final part is summary.

\section{Literature review}

In the application of IoT, wireless sensor is widely used in a variety of monitoring scenarios due to its low price, small size, and strong functions, such as precision agriculture [16-18], environmental monitoring [19-21], and target detection [22-24]. Due to the complex geographical environment of the monitored target area, it is often necessary to use drone to throw a large number of wireless sensors so that the area is completely covered by the sensor. However, the location of wireless sensor placed is random, and it is likely that the perception range of some sensors in the target area is completely covered by its neighbor activation, that is, causing redundant nodes.

In the study of target coverage issue in most WSN $[4,6]$, the randomness of events at coverage points is not taken into account. Only sensor subsets that can cover the target without overlapping in the region where the sensor is densely populated are considered, allowing subset to work alternately to maximize saving energy and extending WSN lifetime. But the problem is that once coverage subset of the sensor is selected, the sensor is always working and certainly causes waste.

Ren et al. [7] considered the interval between events occurrence while ignoring events duration at PoI. The authors assumed that the interval of event columns is independently and identically distributed random variables and these random variables obey exponential distribution with determined parameters, and studied the inactive state scheduling issue of sensor coverage subset. Cheng et al. [9] calculated the probability of capturing event and solved the problem of sensor covering the duration of entire inactive state interval when the event was captured with a certain probability. Dai et al. (2015) [14] took the interval between events and the duration of events at PoI into consideration. The authors assumed that the interval of event column and event duration at PoI is independent and identically distributed sequences of random variables that obey exponential distribution with determined parameters. In addition, Le and Liu [25] studied the capacity of hybrid wireless networks with opportunistic routing. Pei et al. [26] studied the trade-off between security and performance of several recent top performing lightweight block ciphers for the demand of resource-constrained industrial wireless sensor networks. Chu et al. [27] proposed a new scheme of identifying the correctness data scheme for aggregating data in cluster heads in hierarchical WSN based on naive Bayes classification.

Unlike previous literature, this paper discusses on-line adjustable sensor periodic schedule problem in synchronous WSN where the distribution parameters of random variables are not determined. Moreover, by conducting simulation experiments, it is proved that the on-line scheduling scheme in sensor sleep cycle proposed in this paper is more adaptable and energy-saving than off-line scheduling scheme.

\section{Models and assumptions}

This paper assumes that a sufficient amount of sensors are randomly placed into a planar area to capture stochastic events occurred within the target area and events 
randomly occurring at a given PoI. We use Boolean network model [28] to analyze whether a stochastic event is captured by the sensor: if the distance between the location of the activated sensor and the location of PoI where the stochastic event occurs is less than the perception radius $r$ of sensor, the stochastic event is captured by the sensor. Otherwise, it cannot be captured by the sensor. The energy consumed by the sensor per unit time active and inactive state is $k 1$ and $k 2$, respectively. The energy consumption of the sensor to change between active/inactive states is $c$. The average arrival rate of stochastic events of PoI is $v$. In a single scheduling period, a single sensor has two state transitions, and the sensor can be independently scheduled to switch. The duration $X$ and the interval time $Y$ of different stochastic events are independently and identically distributed. Stochastic events at different PoI are also independent. The symbols used in this article and their meanings are shown in Table 1.

\subsection{Prior distribution of stochastic events}

Unlike off-line scheduling scheme, this paper first gives Bayesian estimation of duration and interval distribution parameter describing the randomness of event.

Lemma 1 Stochastic event duration variable $X$ obeys exponential distribution with parameter $\theta^{-1}$, and its probability density function is:

$$
P(X \mid \theta)=\theta^{-1} e^{-X \theta^{-1}}, X>0, E(x)=\theta
$$

where $\theta>0$ is average duration of stochastic events.

Lemma 2 Stochastic event interval variable $Y$ obeys exponential distribution with parameter $\beta^{-1}$, and its probability density function is:

$$
P(Y \mid \beta)=\beta^{-1} e^{-Y \beta^{-1}}, Y>0, E(Y)=\beta
$$

where $\beta>0$ is average interval between stochastic events.

In order to obtain the Bayesian estimation of average duration of stochastic events, it is first necessary to determine the prior distribution of average duration of events $\theta$. In Bayesian statistical theory, the inverse Gamma distribution $\operatorname{IG}(\alpha, \lambda)$ is usually regarded as the prior distribution of unknown parameters $\theta$ [29]. The density function of $\operatorname{IG}(\alpha, \lambda)$ is:

Table 1 Variable symbols and their meanings

\begin{tabular}{ll}
\hline Symbol & Meaning \\
\hline$r$ & Sensor perception radius \\
$k_{1}$ & Energy rate of sensor consumed in active state \\
$k_{2}$ & Energy rate of sensor consumed in inactive state \\
$c$ & Energy consumed by the sensor to change between active/inactive states \\
$V$ & Average arrival rate of stochastic events at Pol \\
$X$ & The time of an event arrives Pol and lasts for a stochastic event, that is, the duration of stochastic \\
$Y$ & event \\
& The interval between the of disappearance of previous event and the arrival of latter event at Pol, \\
$P_{C}$ & Capture probability of stochastic events from arrival Pol until disappearance \\
$Q_{E}$ & Energy efficiency of sensor in capturing stochastic events \\
\hline
\end{tabular}




$$
\pi(\theta)=\frac{\lambda^{\alpha}}{\Gamma(\alpha)} \theta^{-(\alpha+1)} \mathrm{e}^{-\lambda \theta^{-1}}, \theta>0
$$

where $\alpha>0, \lambda>0$ are two undetermined parameters. The mathematical expectation $E(\theta)$ and variance $D(\theta)$ are respectively:

$$
\begin{aligned}
& \mathrm{E}(\theta)=\frac{\lambda}{\alpha-1} \\
& \mathrm{D}(\theta)=\frac{\lambda^{2}}{(\alpha-1)^{2}(\alpha-2)}
\end{aligned}
$$

From Eq.(4) and (5), we can get:

$$
\alpha=(E(\theta))^{2} / D(\theta)+2, \lambda=(E(\theta))^{3} / D(\theta)+E(\theta)
$$

We use the following method to estimate parameters $\alpha, \lambda$. Based on exponential distribution of event duration $\theta^{-1}=1$ in He et al. [15], we perform 100 experiment simulations, and each simulation produces observation values of 100 event duration variable $\mathrm{X}$, i.e., $x_{\mathrm{i} 1}, x_{\mathrm{i} 2}, \ldots, x_{\mathrm{i} 100} \mathrm{i}=1,2, \ldots, 100$, where $i$ represents the number of experiments. So we can get 100 observation values of average event duration parameter are $\theta_{1}, \theta_{2}$, ..., $\theta_{100}$, where $\theta_{i}=1 / \overline{X_{i}}, \overline{X_{i}}=\frac{1}{100} \sum_{j=1}^{100} x_{i j}, i, j=1,2, \ldots, 100$.

Therefore, sample mean value $\bar{\theta}$ and sample variance $S_{\theta}^{2}$ of average event duration of are:

$$
\bar{\theta}=\frac{1}{100} \sum_{i=1}^{100} \theta_{i}, S_{\theta}^{2}=\frac{1}{100-1} \sum_{i=1}^{100}\left(\theta_{i}-\bar{\theta}\right)^{2}
$$

Then, make Eq. (7) as the estimated value of expected $E(\theta)$ and variance $D(\theta)$ respectively and substitute it into Eq. (6) to obtain the estimated value of parameter $\alpha$ and $\lambda$ which are:

$$
\hat{\alpha}=\bar{\theta}^{2} / S_{\theta}^{2}+2, \hat{\lambda}=\bar{\theta}^{3} / S_{\theta}^{2}+\bar{\theta}
$$

Substituting Eq. (8) into $\alpha, \lambda$ in Eq. (3), the prior distribution $\pi(\theta)$ of average stochastic events duration can be obtained.

Similarly, the prior distribution of average interval of stochastic events is $\pi(\beta)$.

\subsection{Bayesian posteriori estimate of distribution parameters}

First, an observation value $\theta$ is generated from Eq. (3) prior distribution $\pi(\theta)$, and then an observation value $x_{1}, x_{2}, \ldots, x_{n}$ of the duration of $n$ events is generated from Eq. (1) conditional distribution $P(X \mid \theta)$., where $x_{i}(i=1,2, \ldots, n)$ represents the duration of $i$ th stochastic event. The joint conditional density function of the sample is:

$$
P(X \mid \theta)=\prod_{i=1}^{n} P\left(x_{i} \mid \theta\right)=\prod_{i=1}^{n}\left(\theta^{-1} \times e^{-x_{i} \theta^{-1}}\right)=\theta^{-n} \times \exp \left(-X_{n} \theta^{-1}\right)
$$

where $X_{n}=\sum_{i=1}^{n} x_{i}$ 。

Multiplying the prior distribution $\pi(\theta)$ by the sample distribution $P(X \mid \theta)$, we can get the kernel of posterior distribution: 


$$
\pi(\theta \mid X)=\frac{P(X \mid \theta) \pi(\theta)}{\int_{0}^{+\infty} P(X \mid \theta) \pi(\theta) d \theta} \propto P(X \mid \theta) \pi(\theta) \propto \theta^{-(\hat{\alpha}+n+1)} \times e^{-\left(\hat{\lambda}+X_{n}\right) / \theta}
$$

Since the inverted Gamma distribution is a conjugate distribution, the posterior distribution of inverted Gamma distribution is still inverse Gamma distribution, that is, Eq. (10) is still the kernel of inverted Gamma distribution. Assuming that the loss function is a squared loss function, then Bayes estimation value of $\theta$ is the mean value of posterior distribution $\pi(\theta \mid X)$. From Eqs. (4) and (5), we can get:

$$
\theta_{n}^{\text {Bayes }}=\frac{\hat{\lambda}+X_{n}}{\hat{\alpha}+n-1}
$$

Therefore, Bayes posterior estimated value of exponential distribution parameter $\theta^{-1}$ obeyed by stochastic event duration variable $X$ is $1 / \theta_{n}^{\text {Bayes }}$.

Similarly, Bayes posterior estimated value of exponential distribution parameter $\beta^{-1}$ obeyed by stochastic event duration variable $Y$ is $1 / \beta_{n}^{\text {Bayes }}$.

\subsection{Correction value of Bayesian posteriori estimation of distributed parameters}

This paper first generates observation value of the duration of $n$ stochastic events from Eq. (9). Then, when a new observation value of stochastic event duration is obtained, that is, the observation value of the duration of $n+1$ th event. At this time, it is necessary to use the observation value $x_{1}, x_{2}, \ldots, x_{n}, x_{n+1}$ of the duration of $n+1$ events to obtain the estimated value $\theta_{n+1}^{\text {Bayes }}$ of the duration parameter $\theta$. Here $\theta_{n+1}^{\text {Bayes }}$ is a correction value for $\theta_{n}^{\text {Bayes }}$. From Eq. (10), Bayesian estimate of posterior distribution $\pi(\theta \mid \mathrm{X})$ is:

$$
\pi(\theta \mid \mathrm{X}) \propto \theta^{-(\hat{\alpha}+n+2)} \times e^{-\left(\hat{\lambda}+X_{n+1}\right) / \theta}
$$

Similarly, the correction value of Bayesian estimation of duration parameter of stochastic event at this time is:

$$
\theta_{n+1}^{\text {Bayes }}=\frac{\hat{\lambda}+X_{n+1}}{\hat{\alpha}+n}=\frac{\hat{\lambda}+X_{n+1}}{\frac{\hat{\lambda}+X_{n}}{\theta_{n}^{\text {Bayes }}}+1}
$$

where

$$
X_{n+1}=X_{n}+x_{n+1}
$$

Therefore, the correction value of Bayes posterior estimated value of exponential distribution parameter $\theta^{-1}$ obeyed by stochastic event duration variable $\mathrm{X}$ is: $1 / \theta_{n}^{\text {Bayes }}$.

Similarly, the correction value of Bayes posterior estimated value of exponential distribution parameter $\beta^{-1}$ obeyed by stochastic event duration variable $Y$ is $1 / \beta_{n}^{\text {Bayes }}$.

\section{Event capture performance analysis}

The following section analyzes the probability that each sensor captures stochastic events occurring in PoI and the energy efficiency of the sensor in capturing stochastic events in periodic schedule $(q, p)$ of sensors in synchronous WSN. Synchronous WSN mean that all sensors use the same periodic schedule $(q, p)$ and the active and inactive state of all sensors are synchronized, where $p$ is the length of time the sensor is 
scheduled, $q$ is the length of time the sensor is in active state, and $p-q$ is the length of time the sensor remains inactive.

\subsection{Capture probability of events in Bayesian estimation of distribution parameters}

The following theorem can be obtained according to the Bayesian estimate of distribution parameters in Chapter 3 and He et al .[15].

Theorem 1 From Eq. (1) and (11), the duration variable of stochastic event obeys the exponential distribution with parameter of $1 / \theta_{n}^{\text {Bayes }}$, then the probability density function of $X$ is:

$$
f(X)=\frac{1}{\theta_{n}^{\text {Bayes }}} \exp \left(-X / \theta_{n}^{\text {Bayes }}\right), X>0, \text { mean }=\theta_{n}^{\text {Bayes }}
$$

Theorem 2 Under the periodic schedule of sensors $(q, p)$, the probability of each sensor capturing stochastic events occurring in PoI is:

$$
\mathrm{P}_{c}=\frac{q}{p}+\frac{1-\exp \left(-(p-q) / \theta_{n}^{\text {Bayes }}\right)}{p / \theta_{n}^{\text {Bayes }}}
$$

Proof Stochastic events captured by sensors can be divided into the following two cases:

First, stochastic events arrive within active state $(0, q)$ of sensors, and the probability of being captured at this time is $q / p$.

Second, stochastic events arrive within inactive state $(q, p)$ of sensors and continue until the next active state $(p, p+q)$, where $t$ is the arrival times of events. At this time, the probability of stochastic events being captured at this time is:

$$
\frac{1}{p} \int_{q}^{p} P_{r}(X \geq p-t) d t
$$

Let $T=p-t$ and substitute $t=p-T$ into Eq. (16), we can get:

$$
\frac{1}{p} \int_{0}^{p-q} P_{r}(X \geq t) d t
$$

Therefore, the sum of probability that stochastic events are captured in both cases is:

$$
P_{c}=\frac{q}{p}+\frac{1}{p} \int_{0}^{p-q} P_{r}(X \geq t) d t
$$

From Theorem 1, we can get:

$$
\begin{gathered}
P_{c}=\frac{q}{p}+\frac{1}{p} \int_{0}^{p-q} P_{r}(X \geq t) d t \\
=\frac{q}{p}+\frac{1}{p} \int_{0}^{p-q}\left(\int_{t}^{+\infty}\left(1 / \theta_{n}^{\text {Bayes }}\right) \exp \left(-X / \theta_{n}^{\text {Bayes }}\right) d X\right) d t \\
=\frac{q}{p}+\frac{1}{p} \int_{0}^{p-q} \exp \left(-t / \theta_{n}^{\text {Bayes }}\right) d t \\
=\frac{q}{p}+\frac{1-\exp \left(-(p-q) / \theta_{n}^{\text {Bayes }}\right)}{p / \theta_{n}^{\text {Bayes }}}(19)
\end{gathered}
$$




\subsection{Energy efficiency of capture events under Bayesian estimates of distribution parameters}

It can be seen from Theorem 1 and Theorem 2 that under periodic schedule $(q, p)$, the number of events that arrives each PoI and is captured by sensors is:

$$
\mathrm{Q}=v \times P_{c}
$$

where

$$
v=\frac{1}{\beta_{n}^{\text {Bayes }}+\theta_{n}^{\text {Bayes }}}
$$

The energy consumption rate per sensor is:

$$
E=\frac{k_{1} \times q+k_{2} \times(p-q)+2 c}{p}
$$

Since one sensor may cover multiple PoIs and one PoI may be covered by multiple sensors at the same time, suppose that there are $m_{1}$ sensors covering $m_{2}$ different PoIs in synchronous sensor network, so the number of events that consumes different unit energy captured in the entire synchronous sensor network is:

$$
Q_{E}=\frac{m_{2}}{m_{1}} \times \frac{Q}{E}=\frac{m_{2}}{m_{1}} \times \frac{\theta_{n}^{\text {Bayes }}}{\left(\theta_{n}^{\text {Bayes }}+\beta_{n}^{\text {Bayes }}\right)} \times \frac{1+q / \theta_{n}^{\text {Bayes }}-\exp \left(-(p-q) / \theta_{n}^{\text {Bayes }}\right)}{k_{1} \times q+k_{2} \times(p-q)+2 c}
$$

\section{On-line adjustable periodic schedule}

Based on above analysis, we study on-line scheduling scheme in synchronous WSN. First, the periodic schedule $(q, p)$ of the entire synchronous WSN is regarded as a largescale on-line sleep cycle scheduling $(q, p)$ of the sensor. Among them, the duration of scheduling time $p$ and the duration of active state $q$ can be corrected in real time.

Solving Eq. (15) to obtain nonlinear equation on the probability of capturing stochastic event as the parameter under Bayesian estimate of exponential distribution parameter:

$$
\begin{aligned}
& F_{1}(p)=P_{c}-k-\frac{1-e^{-(p-p \times k) / \theta_{n}^{\text {Bayes }}}}{p / \theta_{n}^{\text {Bayes }}}=0 \\
& \quad P_{c}>0 \\
& \text { s.t. } \quad p>0 \\
& 0<k=\frac{q}{p} \leq 1
\end{aligned}
$$

Solving Eq. (23) to obtain nonlinear equation on the energy efficiency of sensors capturing events as the parameter in Bayesian estimation of exponential distribution parameter: 


$$
\begin{aligned}
& F_{2}(p)=Q_{E}-\frac{m_{2}}{m_{1}} \times \frac{\theta_{n}^{\text {Bays }}}{\left(\theta_{n}^{\text {Bayes }}+\beta_{n}^{\text {Bayes }}\right)} \\
& \times \frac{1+p \times k / \theta_{n}^{\text {Bayes }}-\exp \left(-(p-p \times k) / \theta_{n}^{\text {Bayes }}\right)}{k_{1} \times p \times k+k_{2} \times(p-p \times k)+2 c}=0 \\
& \quad \begin{array}{l}
Q_{E}>0 \\
p>0 \\
\text { s.t. } \quad 0<k=\frac{q}{p} \leq 1
\end{array}
\end{aligned}
$$

Then use the dichotomy to find zero approximation value of Eqs. (24) and (25), that is, the specific steps of on-line adjustable periodic schedule are as follows:

Step 1: For each set of $1 / \theta$ Bayes n, $1 / \beta$ Bayes n, set a $P_{c}$ or $Q_{E}$ value, determine the interval $[p 1, p 2]$ to make sure $F(p 1) \times \mathrm{F}(\mathrm{p} 2)<0$, set accuracy $\varepsilon$.

Step 2: Find the midpoint value $p$ of $[\mathrm{p} 1, \mathrm{p} 2]$.

Step 3: Calculate $F(p)$. If $F(p)=0$, then $\mathrm{p}$ is zero point; if $\mathrm{F}(\mathrm{p} 1) \times \mathrm{F}(\mathrm{p})<0$, then let $p 2$

$=p$; if $F(p) \times F(p 2)<0$, then let $p 1=p$.

Step 4: If $|p 1-p 2|<\varepsilon$, stop calculation, and zero approximation value is $p 1$ or $p 2$.

Otherwise, return to steps $2-4$.

Therefore, based on dichotomy and Eqs. (24) and (25), on-line scheduling scheme of synchronous WSN under different exponential distribution parameters of Bayesian estimates, stochastic event captured probability, and captured event energy efficiency can be obtained.

\section{Simulation experiment}

\subsection{Parameter settings}

A large number of sensors are randomly placed into $20 \times 20 \mathrm{~m}$ target area in the synchronous WSN. Table 2 shows the relevant experimental parameters.

\subsection{Experiment result}

The simulation results of this paper are based on the average of 100 repetitive experiments. It can be obtained from theoretical derivation and experimental simulation that as the number of events $n$ occurring in PoI increases, the correction values of Bayesian posterior estimation of distribution parameters $\theta^{-1}$ and $\beta^{-1}$ are shown in Table 3.

Table 2 Experimental parameter setting

\begin{tabular}{lc}
\hline Parameter & Value \\
\hline Sensor perception radius: $r$ & $1 \mathrm{~m}$ \\
Energy rate of sensor consumed in active state: $k_{1}$ & $2.369 \mathrm{~J} / \mathrm{h}$ \\
Energy rate of sensor consumed in inactive state: $k_{2}$ & $0.17 \mathrm{~J} / \mathrm{h}$ \\
Energy consumed by the sensor to change between active/inactive states: $c$ & $0.05 \mathrm{~J}$ \\
Unit time & $1 \mathrm{~h}$ \\
Sensors covering $m_{1}$ different Pol covered by $m_{2}$ sensors: $\frac{m_{2}}{m_{1}}$ & 1 \\
\hline
\end{tabular}


Table 3 Correction value of distribution parameters

\begin{tabular}{lllllllll}
\hline The number of arrival events & $\boldsymbol{n}$ & $\mathbf{0}$ & $\mathbf{2 0 0}$ & $\mathbf{4 0 0}$ & $\mathbf{6 0 0}$ & $\mathbf{8 0 0}$ & $\mathbf{1 0 0 0}$ & $\mathbf{1 2 0 0}$ \\
\hline Exponential distribution parameter & $\theta^{-1}$ & 1 & 0.972 & 0.964 & 0.975 & 0.996 & 0.984 & 0.991 \\
Exponential distribution parameter & $\beta^{-1}$ & 2 & 0.972 & 1.191 & 1.340 & 1.455 & 1.550 & 1.578 \\
\hline
\end{tabular}

\subsubsection{Experiment 1: the effect of sensor scheduling time on event capture probability in} different duty cycles.

When $\theta^{-1}=1$, as shown in Fig. 1 , in different duty cycles $q / p$, the capturing probability of stochastic events Pc decreases rapidly as the scheduling duration $p$ of sensor in inactive state increases, that is, the probability of missing stochastic events increase. When the scheduling sensor duration remains the same, the probability of stochastic events capture increases rapidly with the increase of duty cycle, thereby effectively reducing the probability of missing stochastic events.

\subsubsection{Experiment 2: the impact of event capture probability on the duration of scheduling and active state in different scheduling schemes.}

When the duty cycle $q / p=0.4$, as shown in Figs. 2 and 3, as the event capture probability Pc gradually increases, the scheduling duration $p$ and the active state duration $q$ of the sensor in on-line and off-line scheduling schemes both decrease quickly. $p$ and $q$ in on-line scheduling scheme change more rapidly compared to that in off-line scheduling scheme. In addition, when the capture probability of stochastic events is the same, $p$ and $q$ in on-line scheduling scheme change with real time compared to that remains

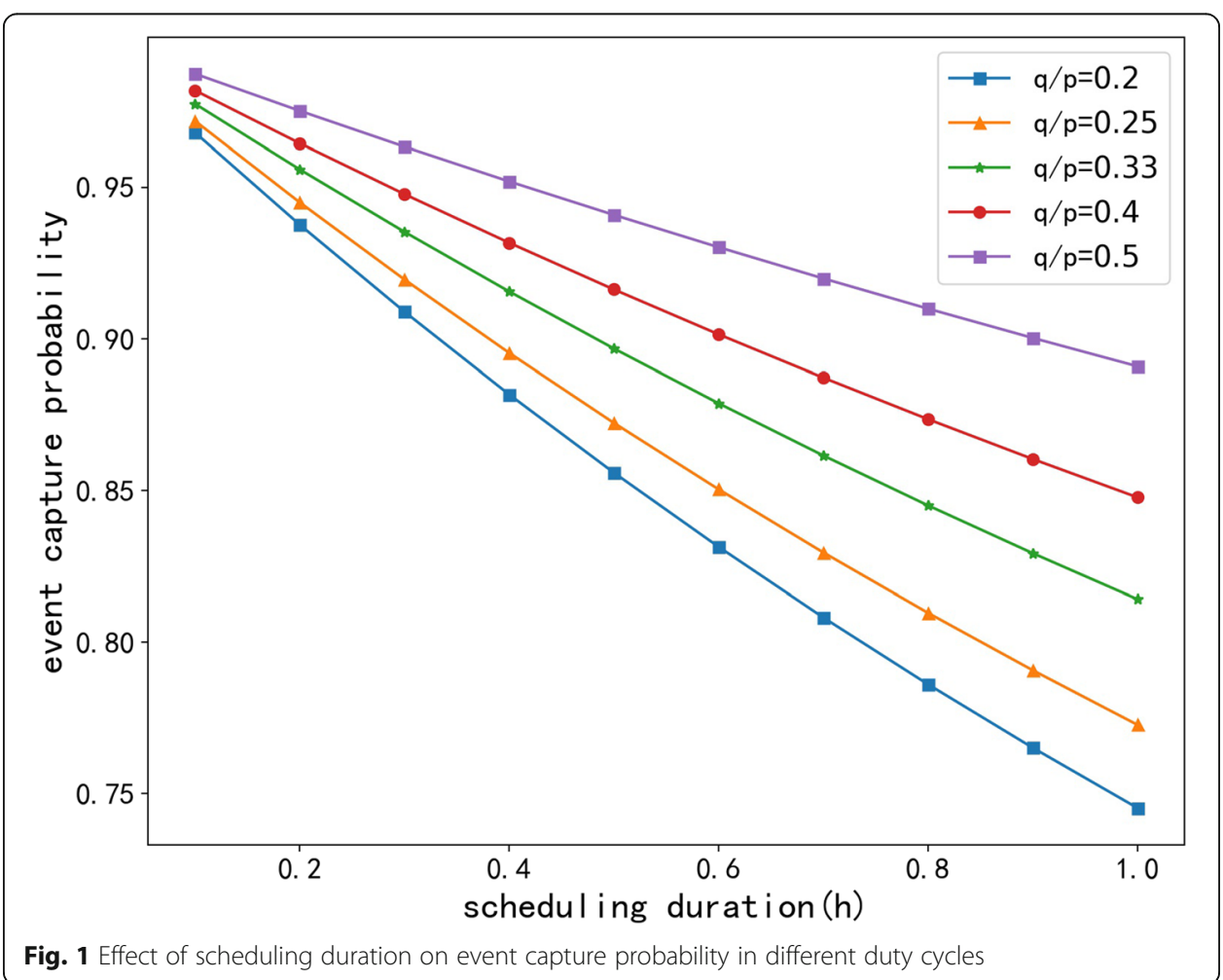




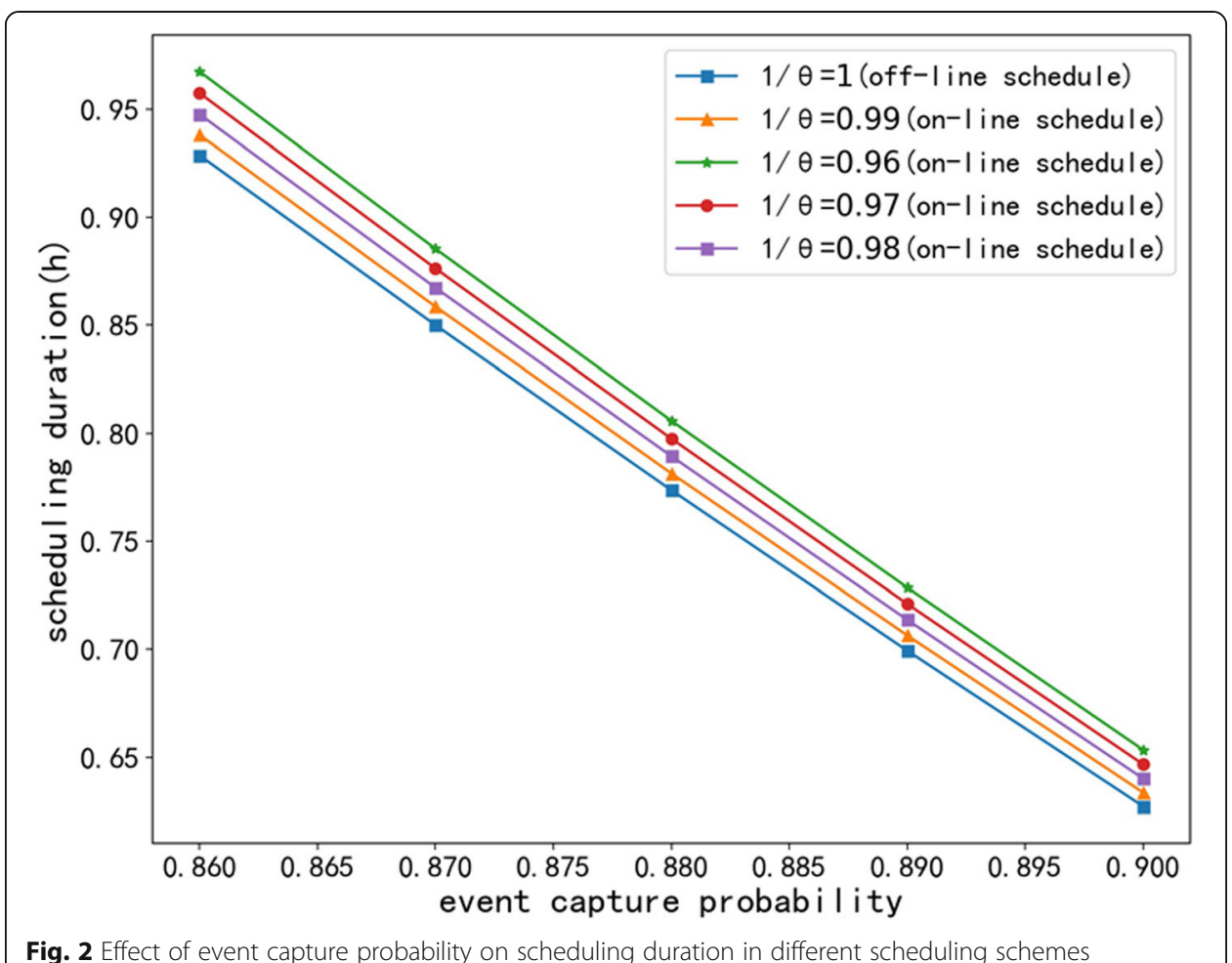

Fig. 2 Effect of event capture probability on scheduling duration in different scheduling schemes

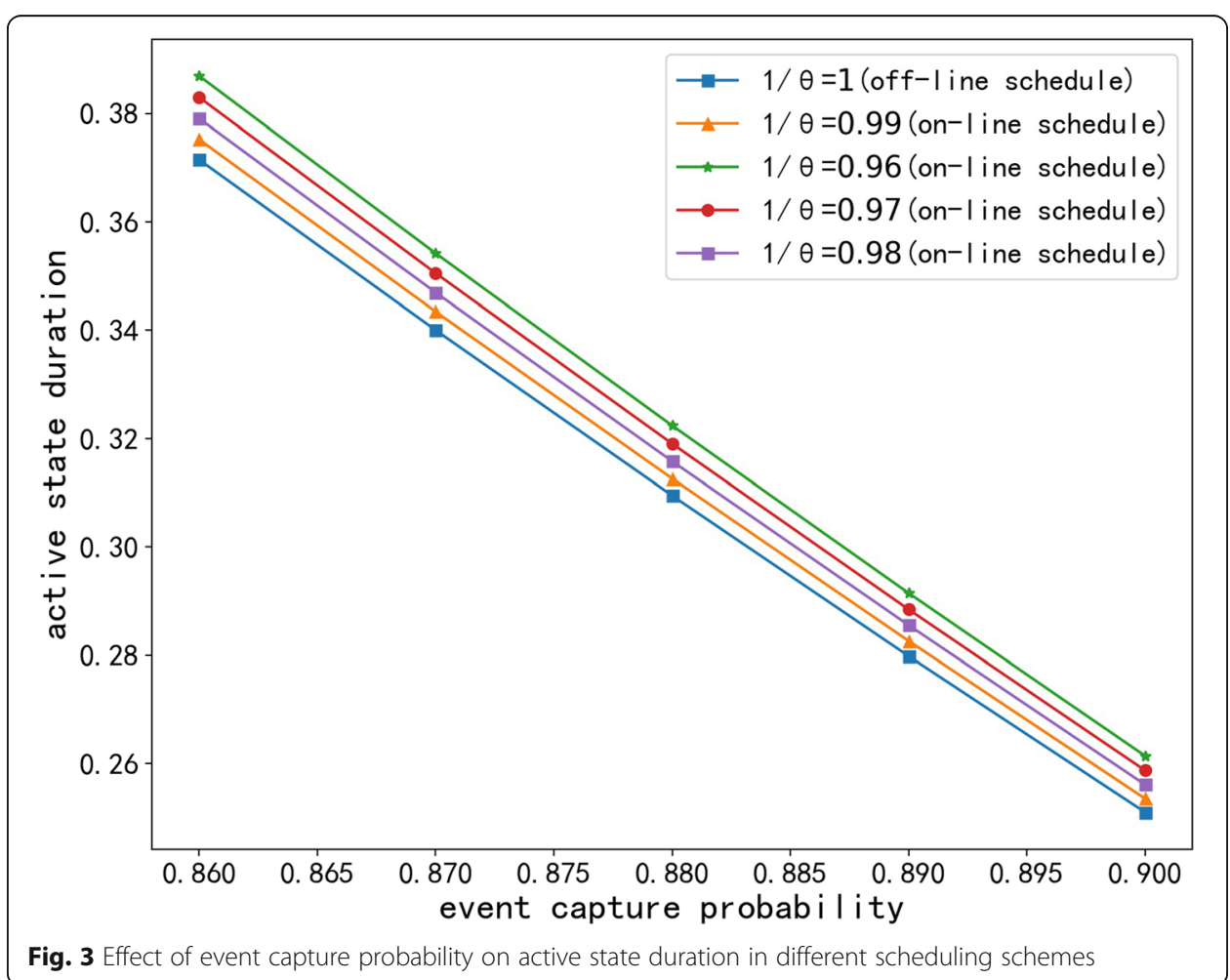


constant in off-line scheduling. The on-line scheduling scheme can be seen as a realtime correction to the off-line scheduling scheme. That is to say, in the same stochastic events capture probability, the on-line scheduling scheme has a longer scheduling duration $p$ and a longer active state duration $q$ compared to that in off-line scheduling scheme, indicating stochastic events that cannot be captured in off-line scheduling scheme can still be captured in the on-line scheduling scheme proposed in this paper, that is, the on-line scheduling scheme can capture more stochastic events.

\subsubsection{Experiment 3: in different scheduling schemes, the effect of energy efficiency of sensor captures event on the duration of scheduling and active state}

When the duty ratio $q / p=0.4$, as shown in Figs. 4 and 5 , as the energy efficiency $Q_{E}$ of sensor capture event gradually increases, the scheduling duration $p$ and active state duration $q$ of the sensor in both scheduling schemes increase rapidly, that is, the sensor active state duration becomes longer $p$ and $q$ in on-line scheduling scheme change more rapidly compared to that in off-line scheduling scheme. In addition, when the energy efficiency is the same, the on-line scheduling scheme is corrected in real time compared with the constant off-line scheduling scheme, that is, $p$ and $q$ are changed with real time. Therefore, we can get the following conclusions: (1) In the same energy efficiency, compared with off-line scheduling scheme, the inactive state $p-q$ becomes longer in on-line scheduling scheme, indicating that on-line scheduling scheme of sensor in inactive state can save more energy. (2) In the same energy efficiency, compared with off-line scheduling scheme, the scheduling active state duration $q$ under the

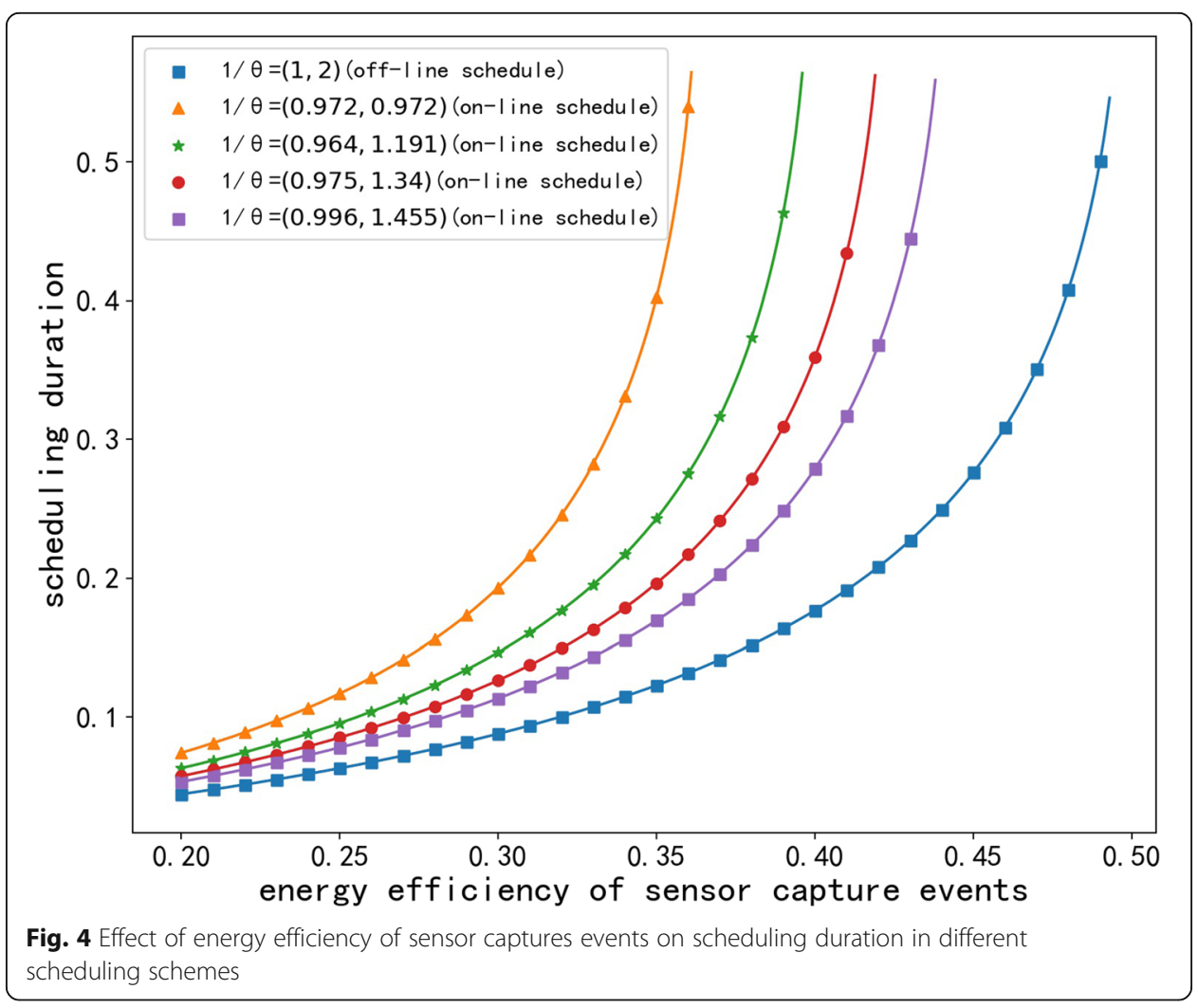




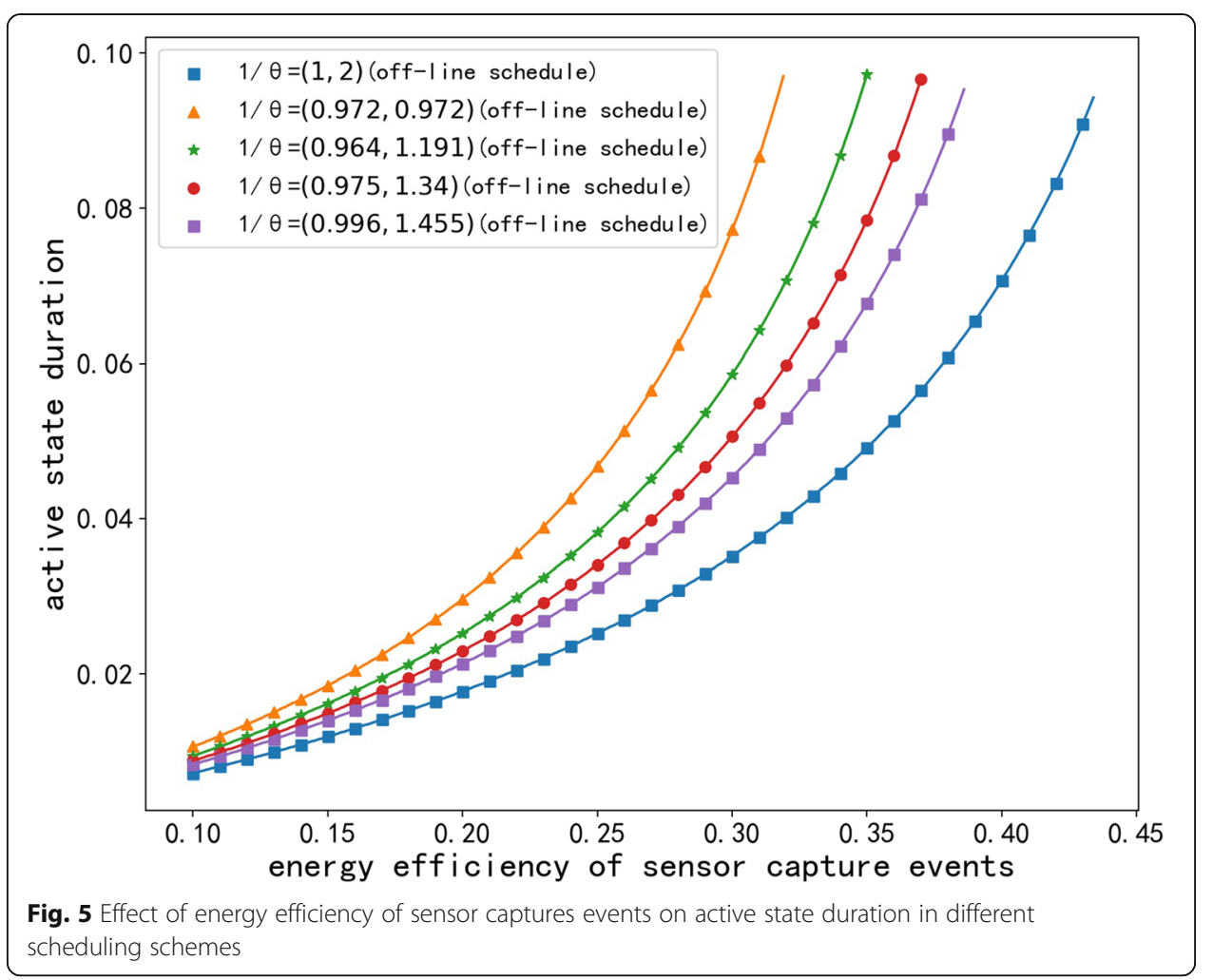

on-line scheduling scheme is longer, indicating that on-line scheduling scheme can capture more stochastic events, thereby further reducing the probability of missing stochastic events, that is, increase the probability of capturing stochastic events.

\section{Conclusion}

This paper studies the on-line scheduling scheme of synchronous WSN. Based on randomness of stochastic events and off-line scheduling scheme of sensors, first we use Bayesian statistical method to make the exponential distribution parameters obey the duration and interval of stochastic events as random variables; then, we used the sample of random variable to get Bayesian estimate of distribution parameter; next we obtain the relationship between periodic schedule of the sensor and Bayesian estimate of distribution parameter; finally, we propose on-line adjustable periodic schedule scheme in synchronous WSN.

In this paper, the on-line scheduling and off-line scheduling schemes are simulated, and experimental results are compared and analyzed. The results show that compared with off-line scheduling scheme, on-line scheduling scheme of sensor in inactive state can reduce the probability of missing stochastic events and increase the probability of capturing events, further saving energy in WSNs and extending network lifetime.

This paper considers that the duration and interval variables of random events are subject to exponential distribution, and future studies can consider that the time variables of random events are subject to more general probability distribution. 
Abbreviations

IOT: Internet of Things; WSN: Wireless sensor networks; Pol: Point of interest

\section{Authors' contributions}

XF built and analyzed the models, designed the experiments, and drafted the manuscript. ZC conceived the study and participated in its design and coordination and helped to draft the manuscript. HT participated in the revision of this paper. All authors read and approved the final manuscript.

\section{Funding}

This work was supported by National Natural Science Foundation of China (no. 71802065, 71831006), Zhejiang Provincial Natural Science Foundation (no. LQ18G010003), and Zhejiang Provincial Philosophy and Social Science Program (no. 18NDJC043YB).

\section{Availability of data and materials}

Data sharing is not applicable to this article as no datasets are generated or analyzed during the current study.

\section{Competing interests}

The authors declare that they have no competing interests.

\section{Author details}

${ }^{1}$ Zhejiang Informatization Development Institute, Hangzhou Dianzi University, Hangzhou 310018, China. ${ }^{2}$ School of Sciences, Hangzhou Dianzi University, Hangzhou 310018, China.

Received: 31 January 2020 Accepted: 1 October 2020

Published online: 14 October 2020

\section{References}

1. X. Li, K. Wang, B. Liu, et al., An improved range-free location algorithm for industrial wireless sensor networks. EURASIP J. Wirel. Commun. Netw. 2020(1), 1 (2020)

2. Zhang Q, Cheng R, Zheng Z. Energy-efficient renewable scheme for rechargeable sensor networks. EURASIP Journal on Wireless Communications and Networking, 2020, 2020(1).

3. Jadidoleslamy $\mathrm{H}$. An introduction to various basic concepts of clustering techniques on wireless sensor networks. Int J Mobile Netw Commun Telematics, 3 1-17, 2013.

4. A. Ahmadi, M. Shojafar, S.F. Hajeforsh, et al., An efficient routing algorithm to preserve k-coverage in wireless sensor networks. J. Supercomput. 68(2), 599-623 (2014)

5. H.P. Gupta, S.V. Rao, T. Venkatesh, Sleep scheduling protocol for k-coverage of three-dimensional heterogeneous WSNs. IEEE Trans. Veh. Technol. 65(10), 8423-8431 (2016)

6. D.S. Hochbaum, A. Pathria, Analysis of the greedy approach in problems of maximum k-coverage. Nav. Res. Logist. 45(6), 615-627 (2015)

7. Z. Ren, P. Cheng, J. Chen, et al., Dynamic activation policies for event capture in rechargeable sensor network. IEEE Trans Parallel Distribut Syst 25(12), 3124-3134 (2014)

8. Wang J, Hu X, Xu X, et al. Event inter-arrival time weighted activation policies for rechargeable wireless sensors. Int Conf Comput Sci Netw Technol IEEE, 2012.

9. P. Cheng, S. He, F. Jiang, et al., Optimal scheduling for quality of monitoring in wireless rechargeable sensor networks. IEEE Trans. Wirel. Commun. 12(6), 3072-3084 (2013)

10. J. Liu, B. Zhao, Z. Ning, Mobile sensors oriented random event capturing. Chin J Sensors Actuators, 257-261 (2014)

11. C. Li, Z. Sun, H. Wang, et al., A novel energy-efficient k-coverage algorithm based on probability driven mechanism of wireless sensor networks. Int J Distribut Sensor Net 1(2), 1-9 (2016)

12. M.M. Islam, M. Ahasanuzzaman, M.A. Razzaque, et al., Target coverage through distributed clustering in directional sensor networks. Eurasip J Wireless Commun Network 2015(1), 167 (2015)

13. J. Tian, M. Gao, G. Ge, Wireless sensor network node optimal coverage based on improved genetic algorithm and binary ant colony algorithm. Eurasip J Wireless Commun Network 2016(1), 104-115 (2016)

14. H. Dai, X. Wu, L. Xu, Practical scheduling for stochastic event capture in energy harvesting sensor networks. Int J Sensor Networks 18(2), 85-96 (2015)

15. S. He, J. Chen, D.K.Y. Yau, et al., Energy-efficient capture of stochastic events under periodic network coverage and coordinated sleep. IEEE Trans Parallel Distribut Syst 23(99), 1090-1102 (2012)

16. M. Srbinovska, C. Gavrovski, V. Dimcev, et al., Environmental parameters monitoring in precision agriculture using wireless sensor networks. J. Clean. Prod. 88, 297-307 (2015)

17. M.H. Anisi, G. Abdul-Salaam, A.H. Abdullah, A survey of wireless sensor network approaches and their energy consumption for monitoring farm fields in precision agriculture. Precis. Agric. 16(2), 216-238 (2015)

18. Nandurkar S R, Thool V R, Thool R C. Design and development of precision agriculture system using wireless sensor network. 2014:1-6.

19. Prabhu B. Environmental monitoring and greenhouse control by distributed sensor network. Int J Adv Netw Appl 2014.

20. J. Paek, J. Hicks, S. Coe, et al., Image-based environmental monitoring sensor application using an embedded wireless sensor network. Sensors 14(9), 15981-16002 (2014)

21. A. Kaushik, R. Kumar, S.K. Arya, et al., Organic-inorganic hybrid nanocomposite-based gas sensors for environmental monitoring. Chem. Rev. 115(11), 4571 (2015)

22. K. Xiao, R. Wang, T. Fu, et al., Divide-and-conquer architecture based collaborative sensing for target monitoring in wireless sensor networks. Inform Fusion 36, 162-171 (2017)

23. H. Mahboubi, W. Masoudimansour, A.G. Aghdam, et al., Maximum lifetime strategy for target monitoring with controlled node mobility in sensor networks with obstacles. IEEE Trans. Autom. Control 61(11), 3493-3508 (2016) 
24. Feng J, Huang $C$, Zhi-Liang $X U$, et al. Research on multi-node cooperative monitoring target and positioning algorithm for wireless sensor networks. Journal of Henan Polytechnic University, 2014-02.

25. T. Le, Y. Liu, On the capacity of hybrid wireless networks with opportunistic routing. EURASIP J. Wirel. Commun. Netw. 2010, 1-9 (2010)

26. C. Pei, Y. Xiao, W. Liang, et al., Trade-off of security and performance of lightweight block ciphers in industrial wireless sensor networks. EURASIP J. Wirel. Commun. Netw. 2018(1), 117 (2018)

27. Chu, S., Dao, T., Pan, J. et al. Identifying correctness data scheme for aggregating data in cluster heads of wireless sensor network based on naive Bayes classification. EURASIP J. Wirel. Commun. Netw.2020, 52 (2020). https://doi.org/https:// doi.org/10.1186/s13638-020-01671-y.

28. S.E. Dallidis, I.G. Karafyllidis, Boolean network model of the Pseudomonas aeruginosa quorum sensing circuits. IEEE Trans Nanobioscience 13(3), 343-349 (2014)

29. T.T. Soong, An extension of the moment method in statistical estimation. SIAM J. Appl. Math. 17(3), 560-568 (1969)

\section{Publisher's Note}

Springer Nature remains neutral with regard to jurisdictional claims in published maps and institutional affiliations.

Submit your manuscript to a SpringerOpen ${ }^{\circ}$ journal and benefit from:

- Convenient online submission

- Rigorous peer review

- Open access: articles freely available online

- High visibility within the field

- Retaining the copyright to your article

Submit your next manuscript at $\boldsymbol{\nabla}$ springeropen.com 